\title{
Modeling and Design Optimization of Near Field Position Sensors
}

\author{
Hubert Zangl' ${ }^{1}$ Lisa-Marie Faller ${ }^{1}$ and Wolfgang Granig ${ }^{2}$ \\ ${ }^{1}$ Institute of Smart System Technologies, Sensors and Actuators, Klagenfurt, Austria, \\ 2 Infineon Technologies AG, Villach, Austria \\ hubert.zangl@aau.at
}

\begin{abstract}
:
A variety of angular position sensors make use of field patterns that are moved or rotated together with an object with respect to a reference. The displacement and/or rotation is then determined using e.g. magnetic field sensors such as Hall sensors or capacitive sensors. These sensors perform a spatial sampling from the field pattern. Based on an appropriate placement of the sensors and a signal processing algorithm, the relative displacement and/or relative rotation should be determined with a low uncertainty. We present an approach for the optimization of the sensor element placement and/or other design parameters based on a meta-model under consideration of correlated uncertainties.
\end{abstract}

Key words: sensor design, optimization, near field sensors

\section{Introduction}

Non-contact measurement of angular positions offers advantages such as low wear, low acoustic noise generation, insensitivity to vibrations and contaminations, etc. Due to the intrinsic robustness, non-contact angular positions sensors based on magnetic sensor elements are very popular. Also capacitive sensors offer such a non-contact operation and have also been demonstrated to work robustly. In such sensors a field pattern is rotated together with a shaft in order to determine the relative angular displacement between the rotor/shaft and the stator. The field pattern can be generated e.g. using a permanent magnet or by modulation of an electric field using a conductive or dielectric rotor. The sensor output is calculated from measurements obtained with individual sensor elements. Illustrations of example sensor principles are shown in Fig. 1. Frequently, the signal obtained for an individual sensor element at a certain location shows an almost sinusoidal variation over the relative angular rotation. The sensor elements are usually placed in such a way, that the obtained signals are phase shifted by 90 degrees, such that an in-phase and a quadrature signal can be determined. Example signals are illustrated in Fig. 2.

In this paper we investigate how we can make an optimal choice for the sensor design and if the commonly used rather intuitive choices for sensor elements is optimal or if better solutions can be found.

\section{Sensor Model and Optimization Parameters}

A simple measurement model for the relation between the signal measured with a sensor element and the relative rotation $\theta$ of a rotor is given by

$$
S(\theta, \eta)=A(\eta,) \sin (\theta-\varphi(\theta))+w
$$

where $S$ is the sensor element output signal, $\theta$ the relative angular position, $A$ the amplitude, and $\varphi$ a phase shift linked to the sensor element location/orientation (compare $[1,2])$. The term $w$ summarizes random deviations and noise. Typically, both the amplitude and the phase shift have to be considered as unknown. Furthermore, also harmonics may be present which requires to extend the model correspondingly. Without such harmonics, at least two sensor elements are required but the actual number of sensor elements is usually higher in order to compensate non-ideal effects. Thus, a measurement vector $\mathbf{S}(\theta, \eta)$, representing the measurements as obtained from several sensor elements located within an integrated circuit or on a printed circuit board (PCB), is obtained. 

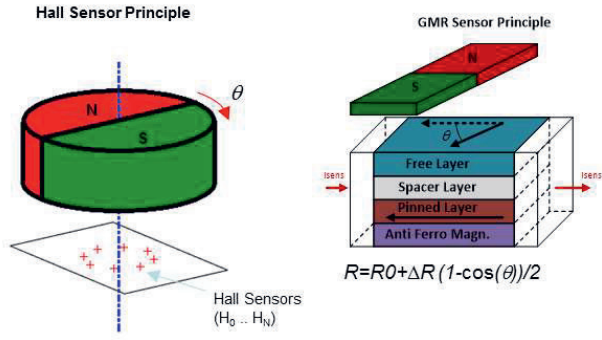

$U_{H 0}=-\Delta U \cos (\theta)$

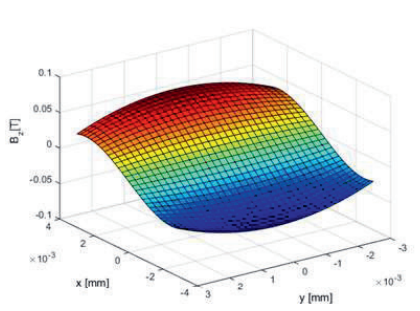

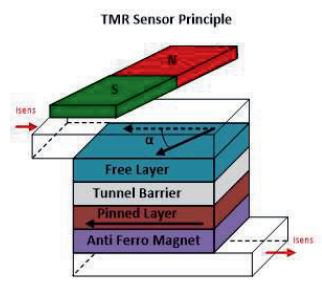

$R=R 0+\Delta R(1-\cos (\theta)) / 2$
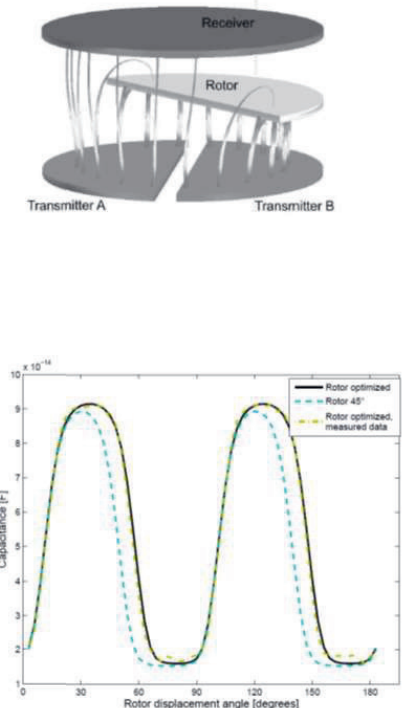

Fig. 1: Examples for angular position sensors. Left: In Hall sensors, the magnetic field strength translates into a proportional voltage signal. When the magnet and thus the field is rotated, an approximately sinusoidal variation is observed near the axis of rotation. Center: In magnetoresisitive sensors such as AMR, GMR and TMR the sensors resistance depends on the angle between a free layer and a reference orientation. As the free layer follows the rotation of the strong external magnet, the resistance changes with the rotation. Bridge circuits are then used to obtain corresponding voltage signals. Right: In capacitive sensors, the field pattern is modulated by a rotor. Depending on the design, the signals may comprise a substantial harmonic contributions, which need to be considered as well.

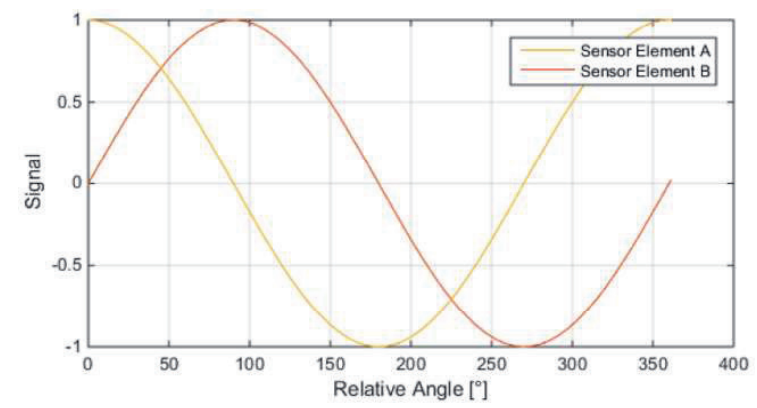

Fig. 2: Example signals as obtained during a rotation for different sensor elements. Depending on e.g. the location of the sensor element or the orientation of a pinned layer fixed layer, the signal gets phase shifted. Having two linearly independent signals, the angle can be determined even when the amplitude of the signals is unknown

The design parameter vector $\boldsymbol{\eta}$ comprises all information describing the configuration, such as the sensor element locations on a silicon die or a PCB, the orientation of the magnetization of a fixed layer, etc. The vector $\mathbf{w}$ represents the random deviations from the ideal model. The contributions to this vector are from random measurement noise but also from other sources, as will be discussed later.

For angular position sensors we suggest a different approach based on the assumption that an accurate determination of the inphase and quadrature (I/Q) contributions will allow for an accurate estimation of the angular position $\theta$. As will be demonstrated, in this case it is possible to actually attain the lower bound - yet for the I and $\mathrm{Q}$ contributions but not directly for the angle $\theta$. Additionally, the computational complexity of the approach is quite low.

The model (1) may be rewritten as

$$
\begin{aligned}
\boldsymbol{S}(\theta, \boldsymbol{\eta})= & I_{A}(\theta, \boldsymbol{\eta}) \cos (\varphi(\boldsymbol{\eta}))+ \\
& +Q_{A}(\theta, \boldsymbol{\eta}) \sin (\varphi(\boldsymbol{\eta}))+w \\
= & \mathbf{H}(\boldsymbol{\eta})\left[\begin{array}{l}
I_{A} \\
Q_{A}
\end{array}\right]+w \\
= & \mathbf{H}(\boldsymbol{\eta}) \boldsymbol{\theta}+w
\end{aligned}
$$

where $I_{A}$ and $Q_{A}$ represent the in-phase and quadrature contributions of the observed measurement vector $\mathbf{S}[6]$. Please note that the individual sensor elements may observe individual amplitudes. However, this may be corrected by individual gain factors, such that common values for $I_{A}$ and $Q_{A}$ can be used as given in [6]. The values for $I_{A}$ and $Q_{A}$ are summarized in the vector $\theta$.

Once we have estimated the $I_{A}$ and $Q_{A}$ contributions, the estimate for the angle $\theta$ is obtained using 


$$
\theta=\operatorname{atan} 2\left(Q_{A}, I_{A}\right)=\operatorname{atan} 2\left(\theta_{2}, \theta_{1}\right)
$$

where atan2(.) is the arctangent function with two arguments, i.e. the arctangent function including the appropriate assignment of the quadrant of the computed angle.

In contrast to a direct optimization for the scalar angle $\theta$, we now have to determine a vector of unknowns. The minimum variance of the estimates of the vector can be obtained from the Fisher Information

$$
\mathbf{I}=\left(\mathbf{H}^{\mathrm{T}} \mathbf{C}^{-1} \mathbf{H}\right)
$$

using

$$
\operatorname{var}\left(\hat{\theta}_{i}\right)=\left[\boldsymbol{I}^{-1}\right]_{i i}
$$

with the optimal estimates

$$
\widehat{\boldsymbol{\theta}}=\left(\mathbf{H}^{\mathrm{T}} \mathbf{C}^{-1} \mathbf{H}\right) \mathbf{H}^{\mathrm{T}} \mathbf{C}^{-1} \mathbf{S}
$$

The matrix $\mathbf{H}$ is defined by the design, adjusting the design parameters will change $\mathbf{H}$ and thus the Fisher Information. Applying e.g. the commonly used D-criterion on the Fisher Information matrix, which maximizes the determinant of $\boldsymbol{I}$, we can minimize the dispersion of the estimates around the true value [9]. The influence of uncertain parameters is summarized in the covariance matrix C. Possible contributions to $\mathbf{C}$ are for example:

- Axes misalignment

- Axial displacement

- Radial displacement

- Inclination

- Magnet Magnetization Defects

- Disturbers

- External fields

- Ferromagnetic, dielectric of conductive materials in the vicinity

- Sensor elements

- Measurement noise

- Offset, gain errors, e.g. due to process variations

- Piezo-resistive effect in ICs

- Piezo-Hall Effect in ICs

While measurement noise is usually considered to lead to uncorrelated deviations of the measurements, other contributions may lead to correlations. For example, piezoresistive [3] and the piezo-Hall effect [4] are related to mechanical stress in integrated circuit. As e.g. the temperature dependent stress is not randomly distributed over the die, also the impact on the measurement will not be randomly distributed. Since the temperature is unknown, it may be considered as random. Consequently, the distribution of stress will lead to a corresponding distribution of stress induced deviations from the nominal behavior. The correlation can be determined experimentally (e.g. [5]), but also using Finite Element Method (FEM) simulation. Fig. 3 shows an example. Clearly, locations that are in vicinity to each other experience similar displacement and similar stress.

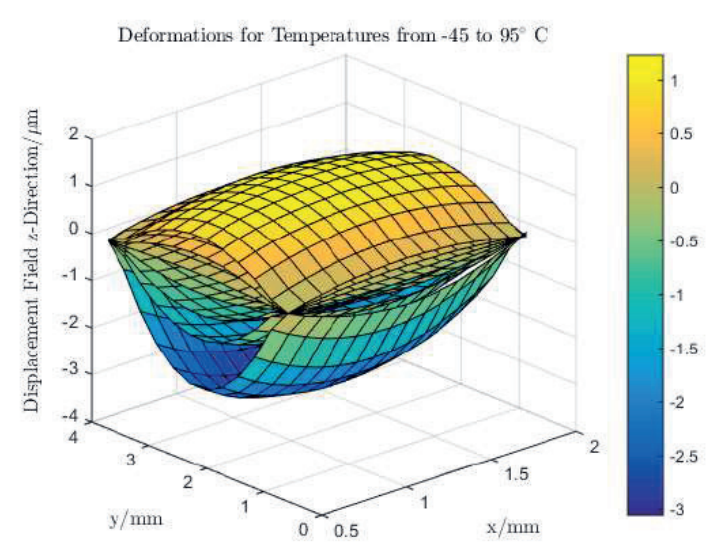

Figure 3: Example for temperature dependent deformations of an integrated circuit within a package as obtained from a FEM simulation. Due to different thermal properties (but also due to process related effects), substantial deformation and stress is present across the circuit. However, the deformations and stresses at close locations are similar.

As a consequence stress induced deviations are subject to spatial correlations. If the temperature is unknown, it might be treated as a random variable. With this approach, also the stress distribution and finally the deviations due to piezoresistive and piezo-Hall effect become random variables.

\section{Optimization Approach and Results}

In general, analytic solutions for the maximization of the determinant of the inverse of the worst case Fisher Information according to

$$
\boldsymbol{\eta}_{\text {opt }}=\arg \max _{\boldsymbol{\eta} \in \boldsymbol{\Gamma}} \min _{\theta \in \Theta} \mathbf{I}(\theta, \boldsymbol{\eta})
$$

are hard to find. Therefore, except for simple cases where an analytic solution can be found, we use a numeric optimization approach. For the results shown in this paper we use the NelderMead algorithm [7] with randomized start vectors in order to find the optimal design.

Different scenarios have been studied. For two sensor elements, a phase shift of $90^{\circ}$ is obtained, for three sensor elements a phase shift of $60^{\circ}$ as expected. However, for more sensor elements, e.g. six elements as shown in Figure 4, a phase shift of $60^{\circ}$ between all magnetic field sensors is not a unique optimum solution. 


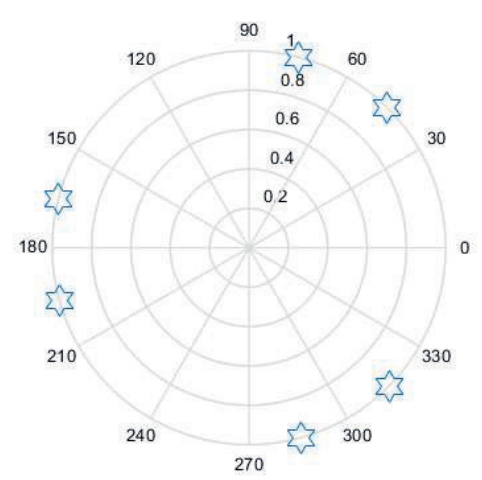

Figure 4: Example solutions for sensor elements (denoted by stars) for uncorrelated deviations but with known harmonics. A non-uniform distribution of the sensor elements is obtained.

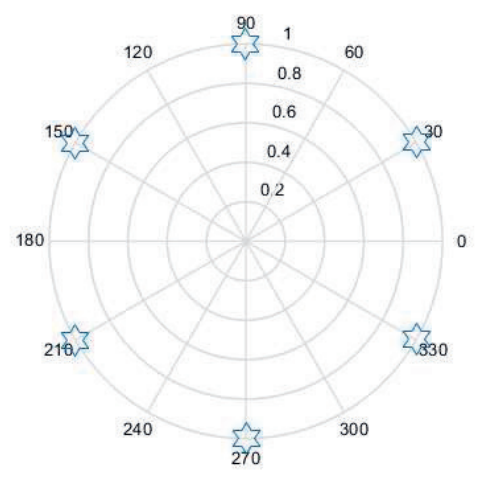

Figure 5: Example solution for six sensor elements for correlated uncertainties. In this case, a uniform distribution of the sensor elements is obtained as the optimal solution.

Further investigations reveal that non-uniform distributions of sensors exist that can achieve a similar performance as uniform distributions also in the case of correlated deviations. As the restriction to use such regular designs can be omitted, new design options are provided, which may allow to make better use of the area of the silicon die.

\section{Conclusion}

We present a fast approach to find D-optimal designs for angular position sensors based on magnetic or electric field patterns. Using metamodels where uncertain parameters are collected in covariance matrices allows to quickly perform the optimization. There is no need to solve field problems during the optimization, but these simulations are only used in the previous step to extract the meta-models and the covariance matrices. Additionally, statistical data from experiments can be considered for both the meta-models and the covariance matrices.

It is shown that the classically used designs are not necessarily optimal. It was also found that non-optimal designs may achieve close-to optimal performance, which opens up the design space and this may be exploited e.g. for a miniaturization of the devices.

\section{References}

[1] U. Ausserlechner, "The optimum layout for giant magnetoresistive angle sensors," IEEE Sensors Journal, vol. 10, no. 10, pp. 1571-1582, Oct. 2010.

[2] H. Zangl and T. Bretterklieber, "Rotor design for capacitive sensors," Proceedings of IEEE Sensors, 2004., 2004, pp. 520-523 vol.1. doi: 10.1109/ICSENS.2004.1426215

[3] Y. Kanda, "Piezoresistance effect of silicon," Sensors and Actuators A: Physical, vol. 28, no. 2, pp. $83-91,1991$.

[4] B. Haelg, "Piezo-hall coefficients of n-type silicon," Journal of Applied Physics, vol. 64, no. 1, pp. 276-282, 1988.

[5] H. Husstedt, U. Ausserlechner, and M. Kaltenbacher, "In-situ analysis of deformation and mechanical stress of packaged silicon dies with an array of hall plates," IEEE Sensors Journal, vol. 11, pp. 2993-3000, Nov 2011.

[6] H. Zangl, Lisa-Marie Faller and W. Granig, "Optimal Design of Angular Position Sensors". IGTE Symposium, September 18 - 21, 2016, Graz, Austria

[7] J. Nelder and R. Mead, "A simplex method for function minimization," Comput. J, vol. 7, pp. 308-313, 1965.

[8] W.Granig, St.Hartmann, Benno Köppl, "Performance and Technology Comparison for GMR versus commonly used Anle Sensor Principles for Automotive Applications", SAE International, 2007

[9] V. Fedorov and P. Hackl, Model-Oriented Design of Experiments, ser. Lecture Notes in Statistics, P. Bickel, P. DIggle, S. Fienberg, K. Krickeberg, N. W. I. Olkin, and S. Zeger, Eds. NY, USA: Springer, 1997, vol. 125. 